\title{
Opportunities for Stewardship in the Transition From Intravenous to Enteral Antibiotics in Hospitalized Pediatric Patients
}

\author{
Jillian M Cotter, MD, MSCS ${ }^{1 *}$, Matt Hall, PhD², Sonya Tang Girdwood, MD, PhD³, John R Stephens, MD4, \\ Jessica L Markham, MD, MSc ${ }^{5}$, James C Gay, MD, MMHC ${ }^{6}$, Samir S Shah, MD, MSCE ${ }^{3}$
}

\begin{abstract}
${ }^{1}$ Children's Hospital Colorado, University of Colorado School of Medicine, Aurora, Colorado; ${ }^{2}$ Children's Hospital Association, Lenexa, Kansas; ${ }^{3}$ Cincinnati Children's Hospital Medical Center and the University of Cincinnati College of Medicine, Cincinnati, Ohio; ${ }^{4}$ North Carolina Children's Hospital, University of North Carolina School of Medicine, Chapel Hill, North Carolina; ${ }^{5}$ Children's Mercy Kansas City, University of Missouri Kansas City (Kansas City, MO); ${ }^{\circ}$ Monroe Carell Children's Hospital at Vanderbilt, Nashville, Tennessee.
\end{abstract}

BACKGROUND/OBJECTIVE: Pediatric patients hospitalized with bacterial infections often receive intravenous (IV) antibiotics. Early transition to enteral antibiotics can reduce hospital duration, cost, and complications. We aimed to identify opportunities to transition from IV to enteral antibiotics, describe variation of transition among hospitals, and evaluate feasibility of novel stewardship metrics.

METHODS: This multisite retrospective cohort study used the Pediatric Health Information System to identify pediatric patients hospitalized with pneumonia, neck infection, orbital infection, urinary tract infection (UTI), osteomyelitis, septic arthritis, or skin and soft tissue infection (SSTI) between 2017 and 2018. Opportunity days were defined as days on which patients received both IV antibiotics and enteral medications, suggesting enteral tolerance. Percent opportunity was defined as opportunity days divided by days on any antibiotics. Both outcomes excluded IV antibiotics that have no alternative oral formulation. We evaluated outcomes per infection and antibiotic and assessed across-hospital variation.
RESULTS: We identified 88,522 aggregate opportunity days in 100,103 hospitalizations. On 57\% of the antibiotic days, there was an opportunity to switch patients to enteral therapy, with greatest opportunity days in SSTI, neck infection, and pneumonia encounters, and with clindamycin, ceftriaxone, and ampicillin-sulbactam. Percent opportunity varied by infection (73\% in septic arthritis to $40 \%$ in pneumonia). There was significant across-hospital variation in percent opportunity for all infections.

CONCLUSION: This multicenter study demonstrated the potential opportunity to transition from IV to enteral therapy in over half of antibiotic days. Opportunity varied by infection, antibiotic, and hospital. Across-hospital variation demonstrated likely missed opportunities for earlier transition and the need to define optimal transition times. Stewardship efforts promoting earlier transition for highly bioavailable antibiotics could reduce healthcare utilization and promote high-value care. We identified feasible stewardship metrics. Journal of Hospital Medicine 2021;16:70-76. (c) 2021 Society of Hospital Medicine acterial infections are a common reason for pediatric hospital admissions in the United States. ${ }^{1}$ Antibiotics are the mainstay of treatment, and whether to administer them intravenously (IV) or enterally is an important and, at times, challenging decision. Not all hospitalized patients with infections require IV antibiotics, and safe, effective early transitions to enteral therapy have been described for numerous infections. ${ }^{2-7}$ However, guidelines describing the ideal initial route of antibiotic administration and when to transition to oral therapy are lacking. 5,7,8 This lack of high-quality evidence-based guidance may contribute to overuse of IV antibiotics for many hospitalized pediatric patients, even when safe and effective enteral options exist. ${ }^{9}$

\footnotetext{
*Corresponding Author: Jillian M. Cotter, MD, MSCS; Email: jillian.cotter@ childrenscolorado.org; Telephone: 720-777-5241; Twitter: jillianmcotter.

Published online first January 20, 2021.

Find additional supporting information in the online version of this article.

Received: July 2, 2020; Revised: September 16, 2020;

Accepted: September 18, 2020

๑) 2021 Society of Hospital Medicine DOI 10.12788/jhm.3538
}

Significant costs and harms are associated with the use of IV antibiotics. In particular, studies have demonstrated longer length of stay (LOS), increased costs, and worsened pain or anxiety related to complications (eg, phlebitis, extravasation injury, thrombosis, catheter-associated bloodstream infections) associated with IV antibiotics. ${ }^{3,4,10-13}$ Earlier transition to enteral therapy, however, can mitigate these increased risks and costs.

The Centers for Disease Control and Prevention lists the transition from IV to oral antibiotics as a key stewardship intervention for improving antibiotic use. ${ }^{14}$ The Infectious Diseases Society of America (IDSA) antibiotic stewardship program guidelines strongly recommend the timely conversion from IV to oral antibiotics, stating that efforts focusing on this transition should be integrated into routine practice. ${ }^{15}$ There are a few metrics in the literature to measure this intervention, but none is universally used, and a modified delphi process could not reach consensus on IV-to-oral transition metrics. ${ }^{16}$

Few studies describe the opportunity to transition to enteral antibiotics in hospitalized patients with common bacterial infections or explore variation across hospitals. It is critical to understand current practice of antibiotic administration in 
order to identify opportunities to optimize patient outcomes and promote high-value care. Furthermore, few studies have evaluated the feasibility of IV-to-oral transition metrics using an administrative database. Thus, the aims of this study were to (1) determine opportunities to transition from IV to enteral antibiotics for pediatric patients hospitalized with common bacterial infections based on their ability to tolerate other enteral medications, (2) describe variation in transition practices among children's hospitals, and (3) evaluate the feasibility of novel IV-to-oral transition metrics using an administrative database to inform stewardship efforts.

\section{METHODS}

\section{Study Design and Setting}

This multicenter, retrospective cohort study used data from the Pediatric Health Information System (PHIS), an administrative and billing database containing encounter-level data from 52 tertiary care pediatric hospitals across the United States affiliated with the Children's Hospital Association (Lenexa, Kansas). Hospitals submit encounter-level data, including demographics, medications, and diagnoses based on International Classification of Disease, Tenth Revision, Clinical Modification (ICD-10-CM) codes. Data were de-identified at the time of submission, and data quality and reliability were assured by joint efforts between the Children's Hospital Association and participating hospitals.

\section{Study Population}

This study included pediatric patients aged 60 days to 18 years who were hospitalized (inpatient or observation status) at one of the participating hospitals between January 1, 2017, and December 31, 2018, for one of the following seven common bacterial infections: community-acquired pneumonia (CAP), neck infection (superficial and deep), periorbital/orbital infection, urinary tract infection (UTI), osteomyelitis, septic arthritis, or skin and soft tissue infection (SSTI). The diagnosis cohorts were defined based on ICD-10-CM discharge diagnoses adapted from previous studies (Appendix Table 1). 3,17-23 To define a cohort of generally healthy pediatric patients with an acute infection, we excluded patients hospitalized in the intensive care unit, patients with nonhome discharges, and patients with complex chronic conditions. ${ }^{24}$ We also excluded hospitals with incomplete data during the study period $(n=1)$. The Institutional Review Board at Cincinnati Children's Hospital Medical Center determined this study to be non-human-subjects research.

\section{Outcomes}

The primary outcomes were the number of opportunity days and the percent of days with opportunity to transition from IV to enteral therapy. Opportunity days, or days in which there was a potential opportunity to transition from IV to enteral antibiotics, were defined as days patients received only IV antibiotic doses and at least one enteral nonantibiotic medication, suggesting an ability to take enteral medications. ${ }^{13}$ We excluded days patients received IV antibiotics for which there was no enteral alternative (eg, vancomycin, Appendix Table 2). When measuring opportunity, to be conservative (ie, to underestimate rather than overestimate opportunity), we did not count as an opportunity day any day in which patients received both IV and enteral antibiotics. Percent opportunity, or the percent of days patients received antibiotics in which there was potential opportunity to transition from IV to enteral antibiotics, was defined as the number of opportunity days divided by number of inpatient days patients received enteral antibiotics or IV antibiotics with at least one enteral nonantibiotic medication (antibiotic days). Similar to opportunity days, antibiotic days excluded days patients were on IV antibiotics for which there was no enteral alternative. Based on our definition, a lower percent opportunity indicates that a hospital is using enteral antibiotics earlier during the hospitalization (earlier transition), while a higher percent opportunity represents later enteral antibiotic use (later transition).

\section{Statistical Analysis}

Demographic and clinical characteristics were summarized by diagnosis with descriptive statistics, including frequency with percentage, mean with standard deviation, and median with interquartile range (IQR). For each diagnosis, we evaluated aggregate opportunity days (sum of opportunity days among all hospitals), opportunity days per encounter, and aggregate percent opportunity using frequencies, mean with standard deviation, and percentages, respectively. We also calculated aggregate opportunity days for diagnosis-antibiotic combinations. To visually show variation in the percent opportunity across hospitals, we displayed the percent opportunity on a heat map, and evaluated percent opportunity across hospitals using chi-square tests. To compare the variability in the percent opportunity across and within hospitals, we used a generalized linear model with two fixed effects (hospital and diagnosis), and parsed the variability using the sum of squares. We performed a sensitivity analysis and excluded days that patients received antiemetic medications (eg, ondansetron, granisetron, prochlorperazine, promethazine), as these suggest potential intolerance of enteral medications. All statistical analyses were performed using SAS v.9.4 (SAS Institute Inc, Cary, North Carolina) and GraphPad Prism 8.0 (GraphPad Software Inc., San Diego, California), and $P$ values $<.05$ were considered statistically significant.

\section{RESULTS}

During the 2-year study period, 100,103 hospitalizations met our inclusion criteria across 51 hospitals and seven diagnosis categories (Table 1). Diagnosis cohorts ranged in size from 1,462 encounters for septic arthritis to 35,665 encounters for neck infections. Overall, we identified 88,522 aggregate opportunity days on which there was an opportunity to switch from IV to enteral treatment in the majority of participants (percent opportunity, 57\%).

\section{Opportunity by Diagnosis}

The number of opportunity days (aggregate and mean per encounter) and percent opportunity varied by diagnosis (Table 2). 
TABLE 1. Cohort Demographics by Diagnosisa

\begin{tabular}{|c|c|c|c|c|c|c|c|}
\hline $\begin{array}{l}\text { Demographic } \\
\text { Variable }\end{array}$ & CAP & $\begin{array}{l}\text { Periorbital/orbital } \\
\text { infection }\end{array}$ & SSTI & Neck infection & UTI & Osteomyelitis & Septic arthritis \\
\hline No. of discharges & 22,605 & 4,150 & 24,736 & 35,665 & 8,673 & 2,812 & 1,462 \\
\hline \multicolumn{8}{|l|}{ Age, y } \\
\hline$<1$ & 11.1 & 10.2 & 11.5 & 4.2 & 34.4 & 3.9 & 7.4 \\
\hline $1-4$ & 54.8 & 37.8 & 34.0 & 54.1 & 22.5 & 24.5 & 42.9 \\
\hline $5-9$ & 22.4 & 28.5 & 22.6 & 24.5 & 18.5 & 28.3 & 26.8 \\
\hline $10-18$ & 11.7 & 23.5 & 32.0 & 17.1 & 24.6 & 43.2 & 22.9 \\
\hline \multicolumn{8}{|l|}{ Sex } \\
\hline Male & 51.3 & 58.9 & 53.6 & 55.0 & 19.6 & 61.0 & 58.9 \\
\hline Female & 48.6 & 41.1 & 46.3 & 45.0 & 80.3 & 39.0 & 41.1 \\
\hline \multicolumn{8}{|l|}{ Race/Ethnicity ${ }^{b}$} \\
\hline Non-Hispanic White & 45.4 & 48.0 & 47.5 & 49.6 & 49.2 & 53.6 & 55.2 \\
\hline Non-Hispanic Black & 16.8 & 22.9 & 19.0 & 204 & 10.3 & 15.0 & 13.3 \\
\hline Hispanic & 23.6 & 17.9 & 22.3 & 18,1 & 27.8 & 19.0 & 18.9 \\
\hline Asian & 3.7 & 2.7 & 2.7 & 2.1 & 3.3 & 2.6 & 3.5 \\
\hline Other & 10.5 & 8.6 & 8.5 & 9.8 & 9.3 & 9.8 & 9.1 \\
\hline \multicolumn{8}{|l|}{ Payor } \\
\hline Government & 53.9 & 55.4 & 60.7 & 55.7 & 59.2 & 45.8 & 42.3 \\
\hline Private & 42.8 & 40.5 & 35.7 & 40.6 & 36.7 & 50.7 & 54.2 \\
\hline Other & 3.3 & 4.1 & 3.6 & 3.8 & 4.1 & 3.5 & 3.5 \\
\hline \multicolumn{8}{|l|}{ Case Mix Index } \\
\hline Mean (SD) & $0.4(0.3)$ & $0.4(0.3)$ & $0.4(0.3)$ & $0.4(0.2)$ & $0.4(0.2)$ & $1(0.6)$ & $1(0.5)$ \\
\hline \multicolumn{8}{|l|}{ Length of stay, d } \\
\hline Median (IQR) & $2[1,3]$ & $2[1,3]$ & $2[1,2]$ & $1[1,2]$ & $2[1,3]$ & $4[3,6]$ & $4[3,5]$ \\
\hline
\end{tabular}

TABLE 2. Potential Opportunity to Transition to Enteral Antibiotics by Diagnosis

\begin{tabular}{|c|c|c|c|c|c|c|c|}
\hline Opportunity Variable & CAP & Periorbital/orbital infection & SSTI & Neck infection & UTI & Osteomyelitis & Septic arthritis \\
\hline Percent opportunity ${ }^{a}$ & 39.7 & 58.1 & 60.2 & 63.6 & 67.8 & 69.0 & 72.7 \\
\hline Aggregate opportunity days ${ }^{\mathrm{b}}$ & 17,238 & 4,254 & 25,359 & 21,696 & 10,334 & 5,948 & 3,693 \\
\hline Opportunity days per encounter, mean (SD) & $0.9(1.3)$ & $1.3(1.4)$ & $1.3(1.2)$ & $1.3(1.2)$ & $1.5(1.2)$ & $2.5(2.4)$ & $2.8(2.5)$ \\
\hline
\end{tabular}

aPercent opportunity, defined as aggregate percent of days patients received IV or enteral antibiotics that were opportunity days

${ }^{b}$ Opportunity days, defined as days patients received IV antibiotics and an enteral nonantibiotic medication

Abbreviations: CAP, community-acquired pneumonia; SSTI, skin and soft tissue infection; UTI, urinary tract infection.

The aggregate number of opportunity days ranged from 3,693 in patients with septic arthritis to 25,359 in patients with SSTI, and mean opportunity days per encounter ranged from 0.9 in CAP to 2.8 in septic arthritis. Percent opportunity was highest for septic arthritis at $72.7 \%$ and lowest for CAP at $39.7 \%$.

\section{Variation in Opportunity Among Hospitals}

The variation in the percent opportunity across hospitals was statistically significant for all diagnoses (Figure). Within hospitals, we observed similar practice patterns across diagnoses. For example, hospitals with a higher percent opportunity for one diagnosis tended to have higher percent opportunity for the other diagnoses (as noted in the top portion of the Figure), and those with lower percent opportunity for one diagnosis tended to also have lower percent opportunity for the other diagnoses studied (as noted in the bottom portion of the Figure). When evaluating variability in the percent opportunity, $45 \%$ of the variability was attributable to the hospital-effect and $35 \%$ to the diagnosis; the remainder was unexplained variability. Sensitivity analysis excluding days when patients received an antiemetic medication yielded no differences in our results.

\section{Opportunity by Antibiotic}

The aggregate number of opportunity days varied by antibiotic (Table 3). Intravenous antibiotics with the largest number of opportunity days included clindamycin $(44,293)$, ceftriaxone 


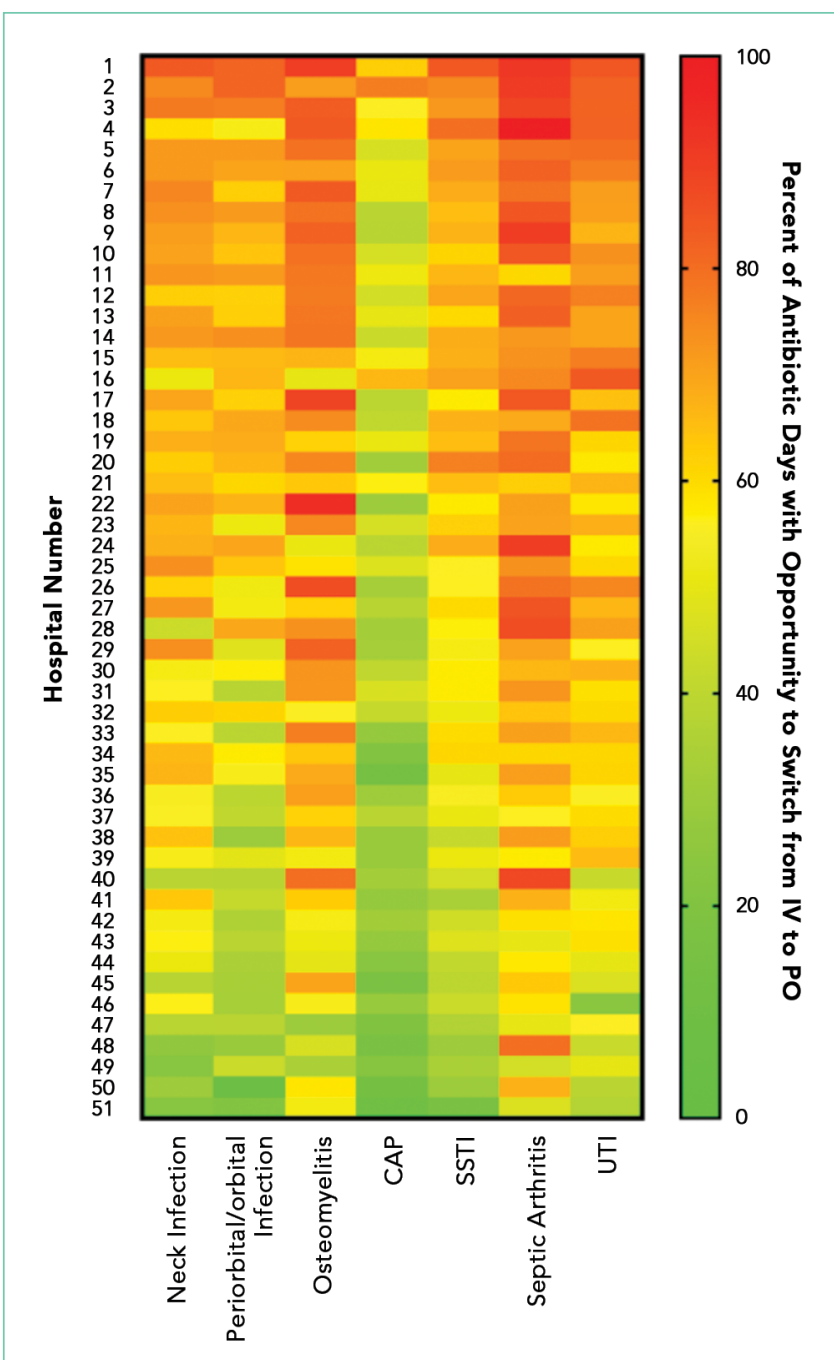

FIG. Heat Map of Percent Opportunity by Diagnosis and Hospital. Hospital-level variation in percent opportunity, or percent of antibiotic days with opportunity to transition from intravenous to enteral antibiotics, are displayed as a heat map. Individual hospitals are displayed in the rows, and diagnoses by column. Hospitals are ordered from highest opportunity (top of map) to lowest opportunity (bottom of map). Color values within each diagnosis correspond to percent opportunity (in order, red representing highest opportunity and green representing lowest opportunity). The variation across hospitals was statistically significant $(P<.001)$ for all diagnoses. Forty-five percent of the variability in percent opportunity was attributable to the hospital-effect and $35 \%$ to the diagnosis-effect.

Abbreviations: CAP, community-acquired pneumonia; SSTI, skin and soft tissue infection; UTI, urinary tract infection.

$(23,896)$, and ampicillin-sulbactam $(15,484)$. Antibiotic-diagnosis combinations with the largest number of opportunity days for each diagnosis included ceftriaxone and ampicillin in CAP; clindamycin in cellulitis, SSTI, and neck infections; ceftriaxone in UTI; and cefazolin in osteomyelitis and septic arthritis.

\section{DISCUSSION}

In this multicenter study of pediatric patients hospitalized with common bacterial infections, there was the potential to transition from IV to enteral treatment in over half of the antibiotic days. The degree of opportunity varied by infection, antibiotic, and hospital. Antibiotics with a large aggregate number of opportunity days for enteral transition included clindamycin, which has excellent bioavailability; and ampicillin and ampicillin-sulbactam, which can achieve pharmacodynamic targets with oral equivalents. ${ }^{25-29}$ The across-hospital variation for a given diagnosis suggests that certain hospitals have strategies in place which permit an earlier transition to enteral antibiotics compared to other institutions in which there were likely missed opportunities to do so. This variability is likely due to limited evidence, emphasizing the need for robust studies to better understand the optimal initial antibiotic route and transition time. Our findings highlight the need for, and large potential impact of, stewardship efforts to promote earlier transition for specific drug targets. This study also demonstrates the feasibility of obtaining two metrics-percent opportunity and opportunity days - from administrative databases to inform stewardship efforts within and across hospitals.

Opportunity days and percent opportunity varied among diagnoses. The variation in aggregate opportunity days was largely a reflection of the number of encounters: Diagnoses such as SSTI, neck infections, and CAP had a large number of both aggregate opportunity days and encounters. The range of opportunity days per encounter (0.9-2.5) suggests potential missed opportunities to transition to enteral antibiotics across all diagnoses (Table 2). The higher opportunity days per encounter in osteomyelitis and septic arthritis may be related to longer LOS and higher percent opportunity. Percent opportunity likely varied among diagnoses due to differences in admission and discharge readiness criteria, diagnostic evaluation, frequency of antibiotic administration, and evidence on the optimal route of initial antibiotics and when to transition to oral formulations. For example, we hypothesize that certain diagnoses, such as osteomyelitis and septic arthritis, have admission and discharge readiness criteria directly tied to the perceived need for IV antibiotics, which may limit in-hospital days on enteral antibiotics and explain the high percent opportunity that we observed. The high percent opportunity seen in musculoskeletal infections also may be due to delays in initiating targeted treatment until culture results were available. Encounters for CAP had the lowest percent opportunity; we hypothesize that this is because admission and discharge readiness may be determined by factors other than the need for IV antibiotics (eg, need for supplemental oxygen), which may increase days on enteral antibiotics and lead to a lower percent opportunity. ${ }^{30}$

Urinary tract infection encounters had a high percent opportunity. As with musculoskeletal infection, this may be related to delays in initiating targeted treatment until culture results became available. Another reason for the high percent opportunity in UTI could be the common use of ceftriaxone, which, dosed every 24 hours, likely reduced the opportunity to transition to enteral antibiotics. There is strong evidence demonstrating no difference in outcomes based on antibiotic routes for UTI, and we would expect this to result in a low percent opportunity. ${ }^{2,31}$ While the observed high opportunity in UTI may relate to an initial unknown diagnosis or concern for systemic infection, this highlights potential opportunities for quality improvement ini- 
TABLE 3. Aggregate Opportunity Days by Intravenous Antibiotic

\begin{tabular}{|c|c|c|c|c|c|c|c|c|}
\hline IV Antibiotic ${ }^{\mathrm{a}}$ & CAP & Periorbital/orbital infection & SSTI & Neck infection & UTI & Osteomyelitis & Septic arthritis & Total opportunity days ${ }^{b}$ \\
\hline Clindamycin & 2,357 & 3,154 & 22,404 & 11,538 & 67 & 3,039 & 1,734 & 44,293 \\
\hline Ampicillin-sulbactam & 812 & 2,038 & 4,848 & 7,504 & 67 & 164 & 51 & 15,484 \\
\hline Ampicillin & 8,734 & 10 & 211 & 596 & 862 & 115 & 170 & 10,698 \\
\hline Azithromycin & 913 & 1 & 15 & 50 & 8 & 0 & 2 & 989 \\
\hline Metronidazole & 22 & 75 & 346 & 36 & 41 & 27 & 4 & 551 \\
\hline Nafcillin & 2 & 30 & 136 & 27 & 1 & 212 & 96 & 504 \\
\hline
\end{tabular}

${ }^{a}$ Top 10 IV antibiotics with largest number of aggregate opportunity days.

bOpportunity days, defined as days patients received IV antibiotics and an enteral nonantibiotic medication.

Abbreviations: CAP, community-acquired pneumonia; IV, intravenous; SSTI, skin and soft tissue infection; UTI, urinary tract infection.

tiatives to promote empiric oral antibiotics in clinically stable patients hospitalized with suspected UTI.

There was substantial variation in percent opportunity across hospitals for a given diagnosis, with less variation across diagnoses for a given hospital. Variation across hospitals but consistency within individual hospitals suggests that some hospitals may promote earlier transition from IV to enteral antibiotics as standard practice for all diagnoses, while other hospitals continue IV antibiotics for the entire hospitalization, highlighting potential missed opportunities at some institutions. While emerging data suggest that traditional long durations of IV antibiotics are not necessary for many infections, the limited evidence on the optimal time to switch to oral antibiotics may have influenced this variation. ${ }^{2-7}$ Many guidelines recommend initial IV antibiotics for hospitalized pediatric patients, but there are few studies comparing IV and enteral therapy. $2,5,9$ Limited evidence leaves significant room for hospital culture, antibiotic stewardship efforts, reimbursement considerations, and/or hospital workflow to influence transition timing and overall opportunity at individual hospitals. ${ }^{7,8,32-34}$ These findings emphasize the importance of research to identify optimal transition time and comparative effectiveness studies to evaluate whether initial IV antibiotics are truly needed for mild —and even severe-disease presentations. Since many patients are admitted for the perceived need for IV antibiotics, earlier use of enteral antibiotics could reduce rates of hospitalizations, LOS, healthcare costs, and resource utilization.

Antibiotics with a high number of opportunity days included clindamycin, ceftriaxone, ampicillin-sublactam, and ampicillin. Our findings are consistent with another study which found that most bioavailable drugs, including clindamycin, were administered via the IV route and accounted for a large number of antibiotic days. ${ }^{35}$ The Infectious Diseases Society of
America recommends that hospitals promote earlier transition to oral formulations for highly bioavailable drugs. ${ }^{7}$ Given the high bioavailability of clindamycin, its common use in highfrequency encounters such as SSTI and neck infections, and the fact that it accounted for a large number of opportunity days, quality improvement initiatives promoting earlier transition to oral clindamycin could have a large impact across health systems. ${ }^{25,26}$ Additionally, although beta-lactam antibiotics such as amoxicillin and amoxicillin-sulbactam are not highly bioavailable, oral dosing can achieve sufficient serum concentrations to reach pharmacodynamic targets for common clinical indications; this could be an important quality improvement initiative. ${ }^{27-29}$ Several single-site studies have successfully implemented quality improvement initiatives to promote earlier IV-to-enteral transition, with resulting reductions in costs and no adverse events noted, highlighting the feasibility and impact of such efforts. ${ }^{13,36-38}$

This study also demonstrates the feasibility of collecting two metrics (percent opportunity and opportunity days) from administrative databases to inform IV-to-oral transition benchmarking and stewardship efforts. While there are several metrics in the literature for evaluating antibiotic transition (eg, days of IV or oral therapy, percentage of antibiotics given via the oral route, time to switch from IV to oral, and acceptance rate of suggested changes to antibiotic route), none are universally used or agreed upon. 15,16,39 The opportunity metrics used in this study have several strengths, including the feasibility of obtaining them from existing databases and the ability to account for intake of other enteral medications; the latter is not evaluated in other metrics. These opportunity metrics can be used together to identify the percent of time in which there is opportunity to transition and total number of days to understand the full extent of potential opportunity for future interventions. As 
demonstrated in this study, these metrics can be measured by diagnosis, antibiotic, or diagnosis-antibiotic combination, and they can be used to evaluate stewardship efforts at a single institution over time or compare efforts across hospitals.

These findings should be interpreted in the context of important limitations. First, we attempted to characterize potential opportunity to transition to enteral medications based on a patient's ability to tolerate nonenteral medications. However, there are other factors that could limit the opportunity to transition that we could not account for with an administrative dataset, including the use of antibiotics prior to admission, disease progression, severity of illness, and malabsorptive concerns. Thus, though we may have overestimated the true opportunity to transition to enteral antibiotics, it is unlikely that this would account for all of the variation in transition times that we observed across hospitals. Second, while our study required patients to have one of seven types of infection, we did not exclude any additional infectious diagnoses (eg, concurrent bacteremia, Clostridioides difficile, otitis media) that could have driven the choice of antibiotic type and modality. Although emerging evidence is supporting earlier transitions to oral therapy, bacteremia is typically treated with IV antibiotics; this may have led to an overestimation of true opportunity. ${ }^{40}$ "Clostridioides" difficile and otitis media are typically treated with enteral therapy; concurrent infections such as these may have led to an underestimation of opportunity given the fact that, based on our definition, the days on which patients received both IV and enteral antibiotics were not counted as opportunity days. Third, because PHIS uses billing days to capture medication use, we were unable to distinguish transitions that occurred early in the day vs those that took place later in the day. This could have led to an underestimation of percent opportunity, particularly for diagnoses with a short LOS; it also likely led to an underestimation of the variability observed across hospitals. Fourth, because we used an administrative dataset, we are unable to understand reasoning behind transitioning time from IV to oral antibiotics, as well as provider, patient, and institutional level factors that influenced these decisions.

\section{CONCLUSION}

Children hospitalized with bacterial infections often receive IV antibiotics, and the timing of transition from IV to enteral antibiotics varies significantly across hospitals. Further research is needed to compare the effectiveness of IV and enteral antibiotics and better define criteria for transition to enteral therapy. We identified ample opportunities for quality improvement initiatives to promote earlier transition, which have the potential to reduce healthcare utilization and promote optimal patient-directed high-value care.

Disclosures: The authors have no conflicts of interest to disclose.

Funding: Dr Tang Girdwood was supported by the National Institute of Child Health and Development Cincinnati Pediatric Clinical Pharmacology Postdoctoral Training Program (5T32HD069054-09) while this work was being conducted.

\section{References}

1. Keren R, Luan X, Localio R, et al. Prioritization of comparative effectiveness research topics in hospital pediatrics. Arch Pediatr Adolesc Med. 2012;166(12):1155-1164. https://doi.org/10.1001/archpediatrics.2012.1266

2. McMullan BJ, Andresen D, Blyth CC, et al. Antibiotic duration and timing of the switch from intravenous to oral route for bacterial infections in children: systematic review and guidelines. Lancet Infect Dis. 2016;16(8):e139-e152. https://doi.org/10.1016/S1473-3099(16)30024-X

3. Keren R, Shah SS, Srivastava R, et al; for the Pediatric Research Inpatient Settings Network. Comparative effectiveness of intravenous vs oral antibiotics for postdischarge treatment of acute osteomyelitis in children. JAMA Pediatr. 2015;169(2):120-128. https://doi.org/10.1001/jamapediatrics.2014.2822

4. Shah SS, Srivastava R, Wu S, et al. Intravenous versus oral antibiotics for postdischarge treatment of complicated pneumonia. Pediatrics. 2016;138(6):e201692. https://doi.org/10.1542/peds.2016-1692

5. Li HK, Agweyu A, English M, Bejon P. An unsupported preference for intravenous antibiotics. PLoS Med. 2015;12(5):e1001825. https://dx.doi. org/10.1371\%2Fjournal.pmed.1001825

6. Dellit TH, Owens RC, McGowan JE Jr, et al; Infectious Diseases Society of America; Society for Healthcare Epidemiology of America. Infectious Diseases Society of America and the Society for Healthcare Epidemiology of America guidelines for developing an institutional program to enhance antimicrobial stewardship. Clin Infect Dis. 2007;44(2):159-177. https://doi. org/10.1086/510393

7. Bradley JS, Byington CL, Shah SS, et al; Pediatric Infectious Diseases Society and the Infectious Diseases Society of America. Management of community-acquired pneumonia (CAP) in infants and children older than 3 months of age: clinical practice guidelines by the Pediatric Infectious Diseases Society and the Infectious Diseases Society of America. Clin Infect Dis. 2011;53(7):e25-e76. https://doi.org/10.1542/peds.2011-2385

8. Septimus EJ, Owens RC Jr. Need and potential of antimicrobial stewardship in community hospitals. Clin Infect Dis. 2011;53 Suppl 1:S8-S14. https://doi. org/10.1093/cid/cir363

9. Schroeder AR, Ralston SL. Intravenous antibiotic durations for common bacterial infections in children: when is enough? J Hosp Med. 2014;9(9):604-609. https://doi.org/10.1002/jhm.2239

10. Christensen EW, Spaulding AB, Pomputius WF, Grapentine SP. Effects of hospital practice patterns for antibiotic administration for pneumonia on hospital lengths of stay and costs. J Pediatric Infect Dis Soc. 2019;8(2):115121. https://doi.org/10.1093/jpids/piy003

11. van Zanten AR, Engelfriet PM, van Dillen K, van Veen M, Nuijten MJ, Polderman $\mathrm{KH}$. Importance of nondrug costs of intravenous antibiotic therapy. Crit Care. 2003;7(6):R184-R190. https://doi.org/10.1186/cc2388

12. Ruebner R, Keren R, Coffin S, Chu J, Horn D, Zaoutis TE. Complications of central venous catheters used for the treatment of acute hematogenous osteomyelitis. Pediatrics. 2006;117(4):1210-1215. https://doi.org/10.1542/ peds.2005-1465

13. Girdwood SCT, Sellas MN, Courter JD, et al. Improving the transition of intravenous to enteral antibiotics in pediatric patients with pneumonia or skin and soft tissue infections. J Hosp Med. 2020;15(1):10-15. https://doi. org/10.12788/jhm.3253

14. Core Elements of Hospital Antibiotic Stewardship Programs. Centers for Disease Control and Prevention. Published 2019. Accessed May 30, 2020. https://www.cdc.gov/antibiotic-use/core-elements/hospital.html

15. Barlam TF, Cosgrove SE, Abbo LM, et al. Implementing an antibiotic stewardship program: guidelines by the Infectious Diseases Society of America and the Society for Healthcare Epidemiology of America. Clin Infect Dis. 2016;62(10):e51-e77. https://doi.org/10.1093/cid/ciw118

16. Science M, Timberlake K, Morris A, Read S, Le Saux N; Groupe Antibiothérapie en Pédiatrie Canada Alliance for Stewardship of Antimicrobials in Pediatrics (GAP Can ASAP). Quality metrics for antimicrobial stewardship programs. Pediatrics. 2019;143(4):e20182372. https://doi.org/10.1542/ peds.2018-2372

17. Tchou MJ, Hall M, Shah SS, et al; Pediatric Research in Inpatient Settings (PRIS) Network. Patterns of electrolyte testing at children's hospitals for common inpatient diagnoses. Pediatrics. 2019;144(1):e20181644. https://doi. org/10.1542/peds.2018-1644

18. Florin TA, French B, Zorc JJ, Alpern ER, Shah SS. Variation in emergency department diagnostic testing and disposition outcomes in pneumonia. Pediatrics. 2013;132(2):237-244. https://doi.org/10.1542/peds.2013-0179

19. Desai S, Shah SS, Hall M, Richardson TE, Thomson JE; Pediatric Research in Inpatient Settings (PRIS) Network. Imaging strategies and outcomes in children hospitalized with cervical lymphadenitis. J Hosp Med. 2020;15(4):197203. https://doi.org/10.12788/jhm.3333 
20. Markham JL, Hall M, Bettenhausen JL, Myers AL, Puls HT, McCulloh RJ. Variation in care and clinical outcomes in children hospitalized with orbital cellulitis. Hosp Pediatr. 2018;8(1):28-35. https://doi.org/10.1542/hpeds.2017-0040

21. Tieder JS, Hall M, Auger KA, et al. Accuracy of administrative billing codes to detect urinary tract infection hospitalizations. Pediatrics. 2011;128(2):323-330. https://doi.org/10.1542/peds.2010-2064

22. Singh JA, Yu S. The burden of septic arthritis on the U.S. inpatient care: a national study. PLoS One. 2017;12(8):e0182577. https://doi.org/10.1371/journal.pone.0182577

23. Foradori DM, Lopez MA, Hall M, et al. Invasive bacterial infections in infants younger than 60 days with skin and soft tissue infections. Pediatr Emerg Care. 2018. https://doi.org/10.1097/pec.0000000000001584

24. Feudtner C, Feinstein JA, Zhong W, Hall M, Dai D. Pediatric complex chronic conditions classification system version 2: updated for ICD-10 and complex medical technology dependence and transplantation. BMC Pediatr. 2014;14:199. https://doi.org/10.1186/1471-2431-14-199

25. Arancibia A, Icarte A, González C, Morasso I. Dose-dependent bioavailability of amoxycillin. Int J Clin Pharmacol Ther Toxicol. 1988;26(6):300-303.

26. Grayson ML, Cosgrove S, Crowe S, et al. Kucers' the Use of Antibiotics: A Clinical Review of Antibacterial, Antifungal, Antiparasitic, and Antiviral Drugs. 7th ed. CRC Press; 2018.

27. Downes KJ, Hahn A, Wiles J, Courter JD, Inks AA. Dose optimisation of antibiotics in children: application of pharmacokinetics/pharmacodynamics in pediatrics'. Int J Antimicrob Agents. 2014;43(3):223-230. https://doi. org/10.1016/j.ijantimicag.2013.11.006

28. Gras-Le Guen C, Boscher C, Godon N, et al. Therapeutic amoxicillin levels achieved with oral administration in term neonates. Eur J Clin Pharmacol. 2007;63(7):657-662. https://doi.org/10.1007/s00228-007-0307-3

29. Sanchez Navarro A. New formulations of amoxicillin/clavulanic acid: a pharmacokinetic and pharmacodynamic review. Clin Pharmacokinet 2005;44(11):1097-1115. https://doi.org/10.2165/00003088-200544110-00001

30. Fine MJ, Hough LJ, Medsger AR, et al. The hospital admission decision for patients with community-acquired pneumonia. Results from the pneumonia Patient Outcomes Research Team cohort study. Arch Intern Med. 1997;157(1):36-44. https://doi.org/10.1001/archinte.1997.00440220040006
31. Pohl A. Modes of administration of antibiotics for symptomatic severe urinary tract infections. Cochrane Database Syst Rev. 2007(4):CD003237. https://doi. org/10.1002/14651858.cd003237.pub2

32. Nageswaran S, Woods CR, Benjamin DK Jr, Givner LB, Shetty AK. Orbital cellulitis in children. Pediatr Infect Dis J. 2006;25(8):695-699. https://doi. org/10.1097/01.inf.0000227820.36036.f1

33. Al-Nammari S, Roberton B, Ferguson C. Towards evidence based emergency medicine: best BETs from the Manchester Royal Infirmary. Should a child with preseptal periorbital cellulitis be treated with intravenous or oral antibiotics? Emerg Med J. 2007;24(2):128-129. https://doi.org/10.1136/ emj.2006.045245

34. Vieira F, Allen SM, Stocks RMS, Thompson JW. Deep neck infection. Otolaryngol Clin North Am. 2008;41(3):459-483, vii. https://doi.org/10.1016/j. otc.2008.01.002

35. Smith M, Shah S, Kronman M, Patel S, Thurm C, Hersh AL. Route of administration for highly orally bioavailable antibiotics. Open Forum Infect Dis. 2017;4(Suppl 1):S498-S499. https://doi.org/10.1093/ofid/ofx163.1291

36. Brady PW, Brinkman WB, Simmons JM, et al. Oral antibiotics at discharge for children with acute osteomyelitis: a rapid cycle improvement project. BMJ Qual Saf. 2014;23(6):499-507. https://doi.org/10.1136/bmjqs-2013-002179

37. Berrevoets $M A H$, Pot JHLW, Houterman AE, et al. An electronic trigger tool to optimise intravenous to oral antibiotic switch: a controlled, interrupted time series study. Antimicrob Resist Infect Control. 2017;6:81. https://doi. org/10.1186/s13756-017-0239-3

38. Fischer MA, Solomon DH, Teich JM, Avorn J. Conversion from intravenous to oral medications: assessment of a computerized intervention for hospitalized patients. Arch Intern Med. 2003;163(21):2585-2589. https://doi.org/10.1001/ archinte.163.21.2585

39. Public Health Ontario. Antimicrobial stewardship programs metric examples. Published 2017. Accessed June 1, 2020. https://www.publichealthontario.ca/-/media/documents/A/2017/asp-metrics-examples.pdf?la=en

40. Desai S, Aronson PL, Shabanova V, et al; Febrile Young Infant Research Collaborative. Parenteral antibiotic therapy duration in young infants with bacteremic urinary tract infections. Pediatrics. 2019;144(3):e20183844. https://doi. org/10.1542/peds.2018-3844 